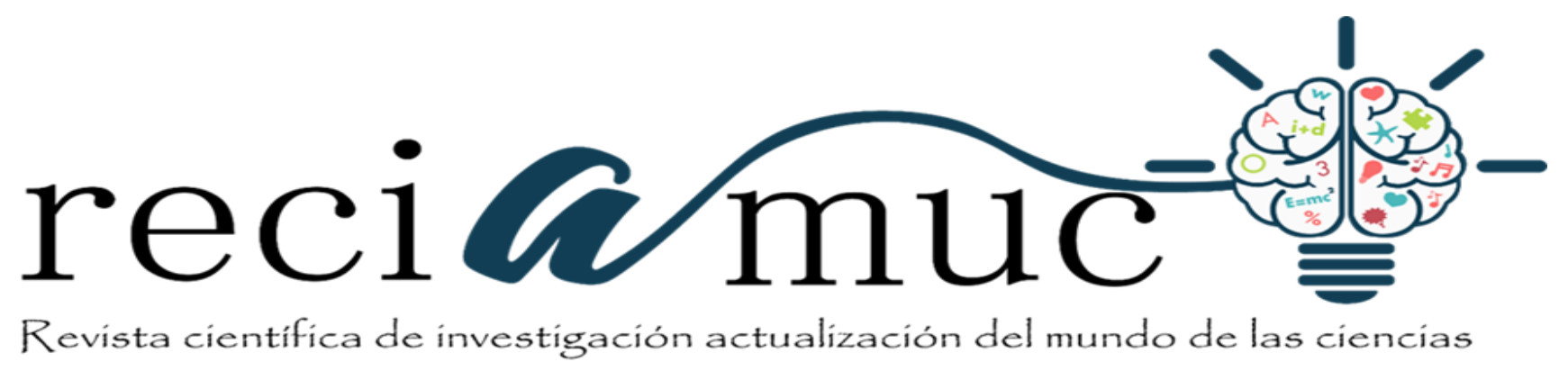

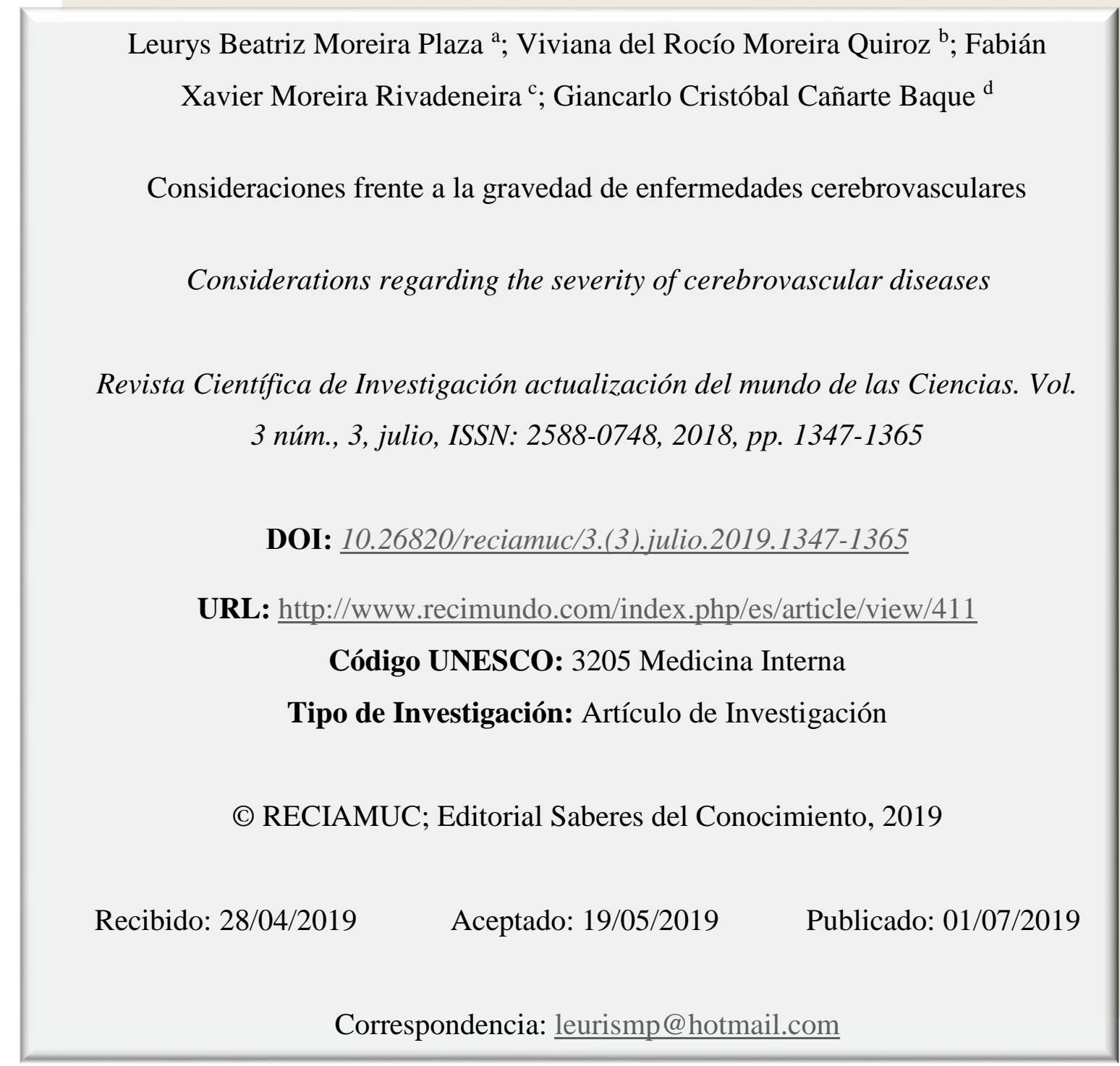

\footnotetext{
a. Médico General Asistencial - Hospital Verdi Cevallos; Portoviejo - Ecuador; leurismp@ hotmail.com;

b. Médico General Asistencial - Hospital Verdi Cevallos; Portoviejo - Ecuador; vivirociomoreira@gmail.com

c. Médico Cirujano; Investigador Independiente; Manta - Ecuador; famori82@ gmail.com

d. Médico General Asistencial Cirugía General; Hospital Verdi Cevallos Balda; Portoviejo - Ecuador; gcanarte@gmail.com
} 


\section{Consideraciones frente a la gravedad de enfermedades cerebrovasculares}

Vol. 3, núm. 3., (2019)

Leurys Beatriz Moreira Plaza; Viviana del Rocío Moreira Quiroz; Fabián Xavier Moreira Rivadeneira; Giancarlo Cristóbal Cañarte Baque

\section{RESUMEN}

El ictus es la segunda causa de muerte y la primera causa de discapacidad, con un coste económico importante. La trombolisis y las unidades ictus son la mayor contribución al tratamiento del accidente cerebrovascular de los últimos años que han cambiado una actitud nihilista hacia una actitud activa, ya que el accidente cerebrovascular es una emergencia médica. El tratamiento con r-TPA ha demostrado ser eficaz en las primeras 3 horas después del accidente cerebrovascular y la FDA y la Agencia Europea del Medicamento (EMEA) ha aprobado su uso. Una gestión protocolizada de la fase aguda para tratar las complicaciones fisiológicas es la Mainstone de unidades de ictus que muestran una reducción en la mortalidad y discapacidad. Estrategias múltiples están en desarrollo con el fin de tratar a más pacientes, los fármacos con una ventana de tiempo terapéutica, trombólisis intravenosa seguido de la trombólisis intraarterial, la selección de los pacientes con falta de correspondencia con las nuevas técnicas de neuroimagen y terapias neuroprotectoras. El tratamiento preventivo también ha experimentado un cambio debido a un mejor conocimiento de la etiopatogenia, el papel de los factores de riesgo vascular y el desarrollo de nuevos fármacos preventivos. El conocimiento de las enfermedades cerebrovasculares es la pista actual para el mejor tratamiento de nuestros pacientes.

Palabras Claves: Enfermedades cerebrovasculares; Trombólisis; Tratamiento. 


\title{
Consideraciones frente a la gravedad de enfermedades cerebrovasculares
}

Vol. 3, núm. 3., (2019)

Leurys Beatriz Moreira Plaza; Viviana del Rocío Moreira Quiroz; Fabián Xavier Moreira Rivadeneira; Giancarlo Cristóbal Cañarte Baque

\begin{abstract}
Stroke is the second cause of death and the first cause of disability with an important economic cost. Thrombolysis and stroke units are the major contribution to stroke treatment of the last years changing a nihilist therapeutic attitude towards an active attitude, stroke is a medical emergency. Treatment with r-TPA has shown to be effective in the first 3 hours following stroke and the FDA and the European Medical Agency (EMEA) have approved its use. A protocolised management of the acute phase to treat physiological complications is the mainstone of stroke units showing a reduction in mortality and disability. Multiple strategies are under development in order to treat more patients, drugs with a longer therapeutic window, intravenous thrombolysis followed by intrarterial thrombolysis, selection of patients with mismatch with new neuroimaging techniques and neuroprotective therapies. Preventive treatment has also experienced a change due to a better knowledge of the ethiopathogeny, the role of vascular risk factors and the development of new preventive drugs. Knowledge of cerebrovascular diseases is today's clue for the best treatment of our patients.
\end{abstract}

Key Words: Cerebrovascular diseases; Thrombolysis; Treatment. 


\section{Consideraciones frente a la gravedad de enfermedades cerebrovasculares}

Vol. 3, núm. 3., (2019)

Leurys Beatriz Moreira Plaza; Viviana del Rocío Moreira Quiroz; Fabián Xavier Moreira

Rivadeneira; Giancarlo Cristóbal Cañarte Baque

\section{Introducción.}

La enfermedad cerebrovascular (ECV) es una de las razones más comunes para las urgencias neurológicas y constituye un grave problema de salud pública. Los datos de la Organización Mundial de la Salud indican que es la segunda causa más común de muerte y la principal causa de discapacidad. En España, es la principal causa de mortalidad específica de la enfermedad en las mujeres. Hasta hace 10 años, los profesionales de la salud y, en particular, los neurólogos han mantenido un conservador en lugar de un enfoque agresivo para esta enfermedad devastadora. Las unidades de ictus y, sobre todo, los métodos para lograr la reperfusión con terapia trombolítica han hecho posible modificar el enfoque: accidente cerebrovascular puede ser una entidad tratable, una circunstancia que mejora significativamente el pronóstico de estos pacientes.

El termino ECV y accidente cerebrovascular se refieren a una alteración en el flujo sanguíneo cerebral que resulta en un transitorio o cambio permanente en la función de 1 o más regiones del cerebro. Hay diferentes tipos de accidente cerebrovascular, dependiendo de la naturaleza de la lesión. El ictus isquémico se debe a una falta de suministro de sangre a un área dada del parénquima cerebral, mientras que apoplejía hemorrágica es causada por la ruptura de un vaso sanguíneo cerebral, con extravasación de sangre en el lecho vascular (Arboix, Álvarez-Sabín, \& Soler, 1998). Los accidentes cerebrovasculares son isquémicos en el $85 \%$ de los casos, y el resto son hemorrágicos. Dados los varios subtipos de accidente cerebrovascular, las variaciones en el perfil de la evolución de la enfermedad, las características topográficas y las diferencias en el mecanismo subyacente y la etiología, hay un número de términos para describir ECV. Es muy importante identificar correctamente el mecanismo causal con el fin de elegir el tratamiento más adecuado y aplicar la prevención secundaria eficaz (Castillo \& Martinez-Vila, 1995).

Dependiendo de la evolución durante las horas iniciales, diferenciamos entre 2 tipos principales de accidentes cerebrovasculares isquémicos: el ataque isquémico transitorio (AIT), clásicamente definido como un déficit neurológico de menos de 24 horas de duración, y el infarto cerebral, con daño irreversible en el cerebro parénquima.

Con respecto a la AIT, cabe señalar que, aunque el término "transitorio" indica una naturaleza benigna, estos episodios se deben considerar una importante señal de advertencia de un infarto cerebral u otras complicaciones cardiovasculares. Aproximadamente $15 \%$ a $30 \%$ de todos $\operatorname{los}$ 


\section{Consideraciones frente a la gravedad de enfermedades cerebrovasculares}

Vol. 3, núm. 3., (2019)

Leurys Beatriz Moreira Plaza; Viviana del Rocío Moreira Quiroz; Fabián Xavier Moreira Rivadeneira; Giancarlo Cristóbal Cañarte Baque

infartos cerebrales están precedidos por un AIT. El diecisiete por ciento de los pacientes que se presentan con infarto cerebral haber tenido un AIT en el mismo día, 9\% en el día anterior y el 43\% durante los siete días anteriores. Además, los pacientes que han sufrido un AIT también tienen un mal pronóstico a largo plazo (Johnston, Gress, Browner, \& Sidney, 2000). Las nuevas técnicas de neuroimagen han hecho posible la detección de una lesión isquémica, es decir, un infarto cerebral, en pacientes cuyos síntomas resuelto dentro de las 24 horas. Estos datos pronósticos y la mejor comprensión de la fisiopatología de la isquemia cerebral han dado lugar a una nueva definición temporal de la AIT como el acontecimiento isquémico que provoca un déficit neurológico que dura menos de una hora y no está asociada con el infarto cerebral en estudios de neuroimagen. Hoy en día, la AIT debe ser considerada una emergencia médica.

El AIT es referido como carotídea, vertebrobasilar o indeterminado, dependiendo del territorio vascular afectado, y, sobre la base de los signos clínicos, puede ser clasificado como retinal (amaurosis fugaz), hemisférico cortical, lacunar, o atípico. Cada una de ellas tiene su propio AIT fisiopatología, cuadro clínico y el pronóstico y, por lo tanto, los tratamientos difieren. En resumen, los pacientes con AIT deben ser considerados un grupo de alto riesgo vascular y, una vez diagnosticado, el mecanismo causal debe ser identificado

Con respecto a infarto cerebral, la causa final es la falta de flujo sanguíneo en alguna parte del cerebro, lo que produce la isquemia y, en última instancia, el infarto (muerte de células del cerebro). La presencia de tejido cerebral isquémico es suficiente para afectar la función cerebral y, por tanto, produce los signos clínicos típicos de accidente cerebrovascular. La región de isquémica, pero no tejido infartado, se conoce como la penumbra isquémica, y es potencialmente recuperable con la terapia de recanalización y el cuidado adecuado. Hoy en día, es posible visualizar el tejido isquémico de penumbra, o falta de coincidencia, por medio de técnicas de neuroimagen, la difusión / perfusión de resonancia magnética (MR), y la tomografía computarizada de perfusión (CT).

\section{Metodología.}

Para el desarrollo de este proceso investigativo, se plantea como metodología la encaminada hacia una orientación científica particular que se encuentra determinada por la necesidad de indagar en forma precisa y coherente una situación, en tal sentido (Davila, 2015) define la metodología "como 


\section{Consideraciones frente a la gravedad de enfermedades cerebrovasculares}

Vol. 3, núm. 3., (2019)

Leurys Beatriz Moreira Plaza; Viviana del Rocío Moreira Quiroz; Fabián Xavier Moreira

Rivadeneira; Giancarlo Cristóbal Cañarte Baque

aquellos pasos previos que son seleccionados por el investigador para lograr resultados favorables que le ayuden a plantear nuevas ideas"'(p.66)

Lo citado por el autor, lleva a entender que el desarrollo de la acción investigativa busca simplemente coordinar acciones enmarcadas en una revisión bibliográfica con el fin de complementar ideas previas relacionadas a las consideraciones en pacientes con enfermedades cerebrovasculares a través de una revisión de literatura, para así finalmente elaborar un cuerpo de consideraciones generales que ayuden a ampliar el interés propuesto.

\section{Tipo de Investigación.}

Dentro de toda práctica investigativa, se precisan acciones de carácter metodológico mediante las cuales, se logra conocer y proyectar los eventos posibles que la determinan, así como las características que hacen del acto científico un proceso interactivo ajustado a una realidad posible de ser interpretada. En este sentido, se puede decir, que la presente investigación corresponde al tipo documental, definido por Castro (2016), "se ocupa del estudio de problemas planteados a nivel teórico, la información requerida para abordarlos se encuentra básicamente en materiales impresos, audiovisuales y /o electrónicos”. (p.41).

En consideración a esta definición, la orientación metodológica permitió la oportunidad de cumplir con una serie de actividades inherentes a la revisión y lectura de diversos documentos donde se encontraron ideas explicitas relacionadas con los tópicos encargados de identificar a cada característica insertada en el estudio. Por lo tanto, se realizaron continuas interpretaciones con el claro propósito de revisar aquellas apreciaciones o investigaciones propuestas por diferentes investigadores relacionadas con el tema de interés, para luego dar la respectiva argumentación a los planteamientos, en función a las necesidades encontradas en la indagación.

\section{Fuentes Documentales.}

El análisis correspondiente a las características que predomina en el tema seleccionado, llevan a incluir diferentes fuentes documentales encargadas de darle el respectivo apoyo y en ese sentido cumplir con la valoración de los hechos a fin de generar nuevos criterios que sirven de referencia a otros procesos investigativos. Para (CASTRO, 2016) las fuentes documentales incorporadas en 


\section{Consideraciones frente a la gravedad de enfermedades cerebrovasculares}

Vol. 3, núm. 3., (2019)

Leurys Beatriz Moreira Plaza; Viviana del Rocío Moreira Quiroz; Fabián Xavier Moreira Rivadeneira; Giancarlo Cristóbal Cañarte Baque

la investigación documental o bibliográfica, "representa la suma de materiales sistemáticos que son revisados en forma rigurosa y profunda para llegar a un análisis del fenómeno".(p.41). Por lo tanto, se procedió a cumplir con la realización de una lectura previa determinada para encontrar aquellos aspectos estrechamente vinculados con el tema, con el fin de explicar mediante un desarrollo las respectivas apreciaciones generales de importancia.

\section{Técnicas para la Recolección de la Información.}

La conducción de la investigación para ser realizada en función a las particularidades que determinan a los estudios documentales, tiene como fin el desarrollo de un conjunto de acciones encargadas de llevar a la selección de técnicas estrechamente vinculadas con las características del estudio. En tal sentido, (Bolívar, 2015), refiere, que es "una técnica particular para aportar ayuda a los procedimientos de selección de las ideas primarias y secundarias”. (p. 71).

Por ello, se procedió a la utilización del subrayado, resúmenes, fichaje, como parte básica para la revisión y selección de los documentos que presentan el contenido teórico. Es decir, que mediante la aplicación de estas técnicas se pudo llegar a recoger informaciones en cuanto a la revisión bibliográfica de los diversos elementos encargados de orientar el proceso de investigación. Tal como lo expresa, (Bolívar, 2015) "las técnicas documentales proporcionan las herramientas esenciales y determinantes para responder a los objetivos formulados y llegar a resultados efectivos" (p. 58). Es decir, para responder con eficiencia a las necesidades investigativas, se introdujeron como técnica de recolección el método inductivo, que hizo posible llevar a cabo una valoración de los hechos de forma particular para llegar a la explicación desde una visión general.

Asimismo, se emplearon las técnicas de análisis de información para la realización de la investigación que fue ejecutada bajo la dinámica de aplicar diversos elementos encargados de determinar el camino a recorrer por el estudio, según, (Bolívar, 2015) las técnicas de procesamiento de datos en los estudios documentales "son las encargadas de ofrecer al investigador la visión o pasos que debe cumplir durante su ejercicio, cada una de ellas debe estar en correspondencia con el nivel a emplear" (p. 123). Esto indica, que para llevar a cabo el procesamiento de los datos obtenidos una vez aplicado las técnicas seleccionadas, tales como: 


\section{Consideraciones frente a la gravedad de enfermedades cerebrovasculares}

Vol. 3, núm. 3., (2019)

Leurys Beatriz Moreira Plaza; Viviana del Rocío Moreira Quiroz; Fabián Xavier Moreira

Rivadeneira; Giancarlo Cristóbal Cañarte Baque

fichas de resumen, textual, registros descriptivos entre otros, los mismos se deben ajustar al nivel que ha sido seleccionado.

\section{Resultados.}

La clasificación etiológica y patogénica de accidente cerebrovascular.

La clasificación etiológica y patogénica de accidente cerebrovascular es esencial para su adecuado tratamiento. Se pueden distinguir 5 subtipos etiológicos y patogénicos que requieren diferentes estrategias de tratamiento: los aterotrombóticos debido a la enfermedad de los vasos grandes, ictus cardioembólico, lacunar por enfermedad de vasos pequeños, que secundaria a una causa poco frecuente (disección arterial, vasculitis, vasoespasmo, etc), y el de etiología indeterminada, o criptogénica, a pesar de un estudio a fondo. Del mismo modo, se distinguen diferentes mecanismos de accidente cerebrovascular. accidente cerebrovascular embólico es el tipo más común, y hay 2 posibles etiologías (cardioembólicas y aterotrombóticos debido a embolia de la arteria a arteria procedentes de una placa ateromatosa), además de la trombosis causada por los cambios de la pared vascular, lipohialinosis en el infarto lacunar,

Los criterios de SSS-PAN se discuten a continuación.

Criterios para infarto aterotrombótica (vaso grande aterosclerosis).

Este diagnóstico requiere el rendimiento de Doppler dúplex y / o un estudio angiográfico (angiografía MR, angiografía CT, o arteriografía) para detectar lesiones de la pared arterial (estenosis y oclusión) en los grandes vasos, tanto extracraneal y supraaórticos, e intracraneal (media, anterior, la arteria cerebral posterior o tronco basilar). Los hallazgos normales, cambios mínimos o el fracaso para llevar a cabo estos estudios descartan el diagnóstico.

\section{Criterios esenciales.}

- Estenosis significativa (mayor del 50\%), oclusión, o una placa ulcerada (más de $2 \mathrm{~mm}$ de espesor) en la arteria intracraneal o arteria extracraneal ipsilateral, demostrada por Doppler dúplex o estudio angiográfico (MR angiografía, o angiografía CT convencional) 


\section{Consideraciones frente a la gravedad de enfermedades cerebrovasculares}

Vol. 3, núm. 3., (2019)

Leurys Beatriz Moreira Plaza; Viviana del Rocío Moreira Quiroz; Fabián Xavier Moreira Rivadeneira; Giancarlo Cristóbal Cañarte Baque

- Ausencia de embolismo cardiogénico o enfermedades del corazón de otra etiología

Otros criterios indicativos de este diagnóstico

1. Los criterios clínicos.

- Presencia de murmullo, ipsilateral al infarto

- Presencia de AIT previo, ipsilateral al infarc

- Antecedentes de enfermedad isquémica del corazón

- Historia de la claudicación intermitente de las extremidades inferiores

2. Criterios de imagen.

- Presencia en CT y / o MR de cortical, o infarto no hemorrágico subcortical medir más de $1,5 \mathrm{~cm}$ en la carótida, o territorio vertebrovasilar

- Estenosis u oclusión del territorio vascular involucrado en la angiografía

Criterios para el ictus isquémico cardioembólico

Criterios esenciales

- La presencia de embolismo cardiogénico

- La presencia de lesiones ateromatosas cerebrovasculares significativas (ver los criterios de infarto atherothombotic) y otras etiologías posibles se debe descartar

En el caso de la enfermedad cardíaca de bajo riesgo (ver la lista de fuentes cardioembólicas), y después de haber descartado otras causas de los accidentes cerebrovasculares, la enfermedad se clasifica como posible ictus cardioembólico.

Otros criterios indicativos de este diagnóstico

1. Los criterios clínicos. 


\section{Consideraciones frente a la gravedad de enfermedades cerebrovasculares}

Vol. 3, núm. 3., (2019)

Leurys Beatriz Moreira Plaza; Viviana del Rocío Moreira Quiroz; Fabián Xavier Moreira Rivadeneira; Giancarlo Cristóbal Cañarte Baque

- déficit neurológico máximo súbita (que se producen en cuestión de segundos o unos pocos minutos)

- El inicio durante el ayuno

- Pérdida de consciencia (transitoria) y / o convulsiones de inicio de síntomas

- Múltiples infartos cerebrales simultáneas

- infartos cerebrales anteriores o AIT en diferentes territorios vasculares

- Historia o coexistencia de embolia sistémica

2. criterios de imagen.

- imágenes por tomografía computarizada que muestran miocardio mayor de 1,5 cm, por lo general cortical, a veces hemorrágica, o múltiples infartos en diferentes territorios vasculares

- evidencia angiográfica de oclusiones angiográficas transitorios, oclusión arterial aislado sin evidencia de lesiones ateroscleróticas, o defecto de llenado central en la porción proximal de una arteria sin cambios ateroscleróticos

Enfermedad de Vaso pequeño (lacunar).

- diámetro del infarto máximo de $1,5 \mathrm{~cm}$, que se encuentra en el territorio arterial o perforar las arteriolas cerebrales (cuyo diámetro es generalmente inferior a 200 micras), debido a lipohialinosis o microatheromatosis de dichos recipientes

- El curso clínico es el de uno de los síndromes lacunares clásicos (hemiparesia puro, síndrome sensorial pura, síndrome sensoriomotor, hemiparesia atáxica, o síndrome mano disartria-torpe)

- La presencia de hipertensión o diabetes mellitus apoya el diagnóstico

- Por definición, no debe haber signos o síntomas corticales

- No debe haber fuentes potenciales de embolia cardíaca o estenosis mayor del $50 \%$ en las arterias extracraneales ipsilaterales 


\section{Consideraciones frente a la gravedad de enfermedades cerebrovasculares}

Vol. 3, núm. 3., (2019)

Leurys Beatriz Moreira Plaza; Viviana del Rocío Moreira Quiroz; Fabián Xavier Moreira Rivadeneira; Giancarlo Cristóbal Cañarte Baque

- La presencia de estenosis mayor del 50\% o placas de ateroma en las arterias de tamaño mediano o grande no descarta la presencia de infarto lacunar

Criterios para el infarto de otras etiologías o causas poco frecuentes.

Esta categoría incluye pacientes con infarto cerebral agudo debido a causas poco frecuentes, tales como enfermedades vasculares ateroscleróticas (inflamatorias, no inflamatoria, infecciosa, hereditaria), estados de hipercoagulabilidad, trastornos hematológicos, migrañamiocardio, vaso espasmo, y otras enfermedades hereditarias y metabólicas.

Las etiologías mencionadas de infarto cerebral cardioembólico y la presencia de aterosclerosis en las arterias extracraneales deben descartarse.

\section{Diagnóstico.}

La rápida identificación de accidente cerebrovascular, la determinación de su etiología y la patogénesis, y su tratamiento adecuado, con terapias específicas (agentes fibrinolíticos) durante la fase aguda, la atención general en las unidades de ictus, y el tratamiento preventivo específicos para cada subtipo de accidente cerebrovascular son vitales en el intento de reducir el daño cerebral irreversible, a prevenir la recurrencia y, así, mejorar la recuperación funcional del paciente.

El proceso de diagnóstico incluye los siguientes elementos: historia clínica, examen físico y neurológico general y pruebas auxiliares.

En la historia clínica, se debe prestar atención a la historia personal y familiar de enfermedad vascular, la ictal, o la aparición súbita de síntomas neurológicos focales y, sobre todo, el tiempo transcurrido desde el inicio con el fin de decidir si se justifica el tratamiento fibrinolítico de emergencia. 


\section{Consideraciones frente a la gravedad de enfermedades cerebrovasculares}

Vol. 3, núm. 3., (2019)

Leurys Beatriz Moreira Plaza; Viviana del Rocío Moreira Quiroz; Fabián Xavier Moreira

Rivadeneira; Giancarlo Cristóbal Cañarte Baque

El examen neurológico debe confirmar la sospecha de focalidad neurológica y hará que sea posible trazar la topografía de la carrera. Estos datos, junto con el examen físico, los hallazgos de laboratorio, electrocardiograma (ECG), ayudarán a establecer la posible etiología de la carrera (soplo carotídeo, la fibrilación auricular en el ECG, cardiomegalia en el pecho ·de rayos, etc.).

Entre los exámenes más específicos, la TC craneal de emergencia es esencial para descartar una hemorragia cerebral y otras causas de signos neurológicos focales, y para confirmar la naturaleza isquémica del proceso. En las primeras horas después de un infarto cerebral, la craneal CT puede ser normal o revelar signos tempranos de infarto que serán de gran utilidad en la valoración de la extensión del infarto e iniciar la terapia fibrinolítica.

Un estudio Doppler transcraneal dúplex que implica troncos supraaórticos se llevará a cabo en todos los pacientes que han sufrido un accidente cerebrovascular isquémico. El estudio de la arteria carótida nos permitirá diagnosticar la enfermedad aterotrombótica subyacente y evaluar tratamientos preventivos específicos, como la endarterectomía carotídea. Doppler transcraneal duplex nos permite diagnosticar estenosis intracraneales, examinar la circulación colateral, confirmar la recanalización arterial después de la administración de la terapia fibrinolítica, e incluso detectar de derecha a izquierda en derivación a través de un foramen oval permeable (PFO) utilizando una solución de microburbujas. Por otro lado, proporciona información valiosa en la evaluación preoperatoria y para el pronóstico de la estenosis carotídea (estudios de la circulación colateral y reserva hemodinámica y la detección de microembolias).

La alta sensibilidad y especificidad del estudio de ultrasonido (dúplex color de los troncos supraaórticos más Doppler dúplex transcraneal) hace que sea posible, mediante la adición de alguna otra técnica de imagen no invasiva como la angiografía por resonancia magnética o angiografía TC, para decidir si realizar o no la endarterectomía, sin la utilización de la angiografía por sustracción digital.

La presencia del potencial de embolismo cardiogénico debe evaluarse sobre la base de la historia clínica, el examen físico, el pecho ·ray, y ECG. Esto hace que sea posible iniciar el tratamiento anticoagulante preventivo. El rendimiento de la ecocardiografía transtorácica se recomienda 


\section{Consideraciones frente a la gravedad de enfermedades cerebrovasculares}

Vol. 3, núm. 3., (2019)

Leurys Beatriz Moreira Plaza; Viviana del Rocío Moreira Quiroz; Fabián Xavier Moreira Rivadeneira; Giancarlo Cristóbal Cañarte Baque

cuando se han detectado exámenes anteriores sin evidencia de enfermedad, cuando se sospecha una etiología cardioembólico y en los accidentes cerebrovasculares en pacientes jóvenes. En aquellos casos en que se sospecha la ateromatosis aórtica y cuando se detecta un cortocircuito de derecha a izquierda, el estudio debe completarse con la ecocardiografía transesofágica.

RM craneal es muy útil en el tratamiento del ictus, no sólo porque ayuda a confirmar y determinar la localización topográfica de los infartos, sino también por su utilidad en el tratamiento agudo.Iimágenes de difusión ponderada (DWI) muestran el tejido infartado en la fase aguda y las imágenes de perfusión ponderada (PWI) hacer que sea posible cuantificar el tejido hipoperfundido; la diferencia entre las 2 zonas identificará el tejido o desajuste de penumbra, que es potencialmente recuperable con la terapia de recanalización. Se recomienda la RM para localizar y confirmar infartos lacunares, y la evaluación de accidente cerebrovascular territorio vertebrobasilar. El estudio debe completarse con angiografía por resonancia magnética o, en casos excepcionales,

\section{Tratamiento.}

El accidente cerebrovascular es una emergencia médica que requiere una intervención inmediata. El infarto cerebral se establece progresivamente durante varias horas, y el tamaño del infarto se puede minimizar si actuamos dentro de esa ventana terapéutica.

En los últimos años, se ha producido un cambio sustancial en el enfoque terapéutico para el ictus isquémico. El concepto de accidente cerebrovascular isquémico como un proceso que progresa en un período de horas ha proporcionado nuevas perspectivas, con las drogas para los agentes de recanalización y neuroprotectores, y con tratamiento médico que es más eficaz en la prevención de un mayor daño neuronal. La evaluación apropiada de la causa de la carrera, su fisiopatología y vascular topografía determinará el tratamiento ideal y, en consecuencia, mejorar el pronóstico. La aplicación de estas medidas diagnósticas y terapéuticas de acuerdo con protocolos existentes dentro de las primeras 6 horas de la aparición de síntomas reduce significativamente la discapacidad y acorta la estancia hospitalaria (Davalos, Castillo, \& Martinez-Vila, 1995) 


\section{Consideraciones frente a la gravedad de enfermedades cerebrovasculares}

Vol. 3, núm. 3., (2019)

Leurys Beatriz Moreira Plaza; Viviana del Rocío Moreira Quiroz; Fabián Xavier Moreira Rivadeneira; Giancarlo Cristóbal Cañarte Baque

El descubrimiento de la terapia trombolítica, junto con la implementación de unidades de ictus (aumento de la vigilancia y el seguimiento durante los primeros días), ha sido el avance más importante en el tratamiento de pacientes con accidente cerebrovascular en los últimos años.

El tratamiento del accidente cerebrovascular se basa en tratamiento agudo y la prevención de recurrencias. El tratamiento agudo se puede dividir en 2 partes: medidas generales y la terapia de recanalización.

Medidas generales: la unidad de ictus.

Cuidado general ha demostrado ser eficaz para mejorar el pronóstico de los pacientes con accidente cerebrovascular. Hipoxia, hipertensión severa, o hipotensión, hiperglucemia, hipotermia, deshidratación y desnutrición son factores que tienen una influencia negativa en el pronóstico funcional en el ictus, ya que provocan mayor daño neuronal (Powers, 2003). La monitorización de los signos vitales y el tratamiento temprano en la detección de estas complicaciones, así como la movilización precoz, son todas las medidas incluidas en las directrices y recomendaciones para la atención al ictus.

Las unidades de ictus consisten en equipos multidisciplinarios, coordinados por neurólogos con formación especializada en las enfermedades cerebrovasculares, la prestación de atención inmediata, con una disponibilidad continua de las técnicas de diagnóstico como la tomografía computarizada craneal, Doppler transcraneal dúplex participación de los troncos supraaórticos y, con frecuencia, la difusión y la perfusión RM, y angiografía por resonancia magnética.

Las unidades de ictus deben estar equipados con 4 a 8 camas, según las necesidades, y proporcionan cuidados intensivos y no intensiva. Allí, los pacientes son monitorizados continuamente para la detección precoz y la corrección de los factores que pueden empeorar el proceso isquémico agudo, y puede recibir un tratamiento específico para el infarto cerebral. En estas unidades, la observación continua será mantenido por el personal de enfermería. se siguió un protocolo normalizado para la evaluación de diagnóstico, el tratamiento y la rehabilitación. Los protocolos también deben adoptarse para la terapia trombolítica, la prevención de las complicaciones de un derrame cerebral (edema cerebral, ataques epilépticos), prevención de 


\section{Consideraciones frente a la gravedad de enfermedades cerebrovasculares}

Vol. 3, núm. 3., (2019)

Leurys Beatriz Moreira Plaza; Viviana del Rocío Moreira Quiroz; Fabián Xavier Moreira Rivadeneira; Giancarlo Cristóbal Cañarte Baque

complicaciones médicas (control de la glucosa en la sangre, la presión sanguínea arterial y la temperatura corporal, la prevención de la trombosis venosa profunda, la detección de arritmias, y las infecciones del tracto respiratorio, la atención nutricional, la prevención de las úlceras de decúbito), y la rehabilitación temprana.

Esta atención especializada multidisciplinaria es la base para el concepto de unidades de ictus. El desarrollo de estas unidades se ha traducido en una reducción significativa en las tasas de mortalidad y morbilidad, la estancia hospitalaria y los costos (Langhorne, Willians, Gilchrist, \& Howie, 2003). Se han encontrado resultados similares, con un mejor pronóstico en los pacientes con accidente cerebrovascular grave con respecto al entorno hospitalario convencional.

\section{Recanalización Terapias: Trombolisis}

\section{La trombolisis intravenosa}

El propósito de la terapia trombolítica es lograr la restauración precoz de flujo arterial y preservar el tejido neuronal reversiblemente dañado de la penumbra isquémica, por medio de un agente relativamente seguro, con el fin de mejorar el curso de la enfermedad. Menor daño neuronal en la incapacidad funcional menos grave.

La Administración de Alimentos y Medicamentos de Estados Unidos (FDA) aprobó el uso del activador del plasminógeno tisular recombinante (rt-PA) como tratamiento de primera línea para el accidente cerebrovascular isquémico en junio de 1998, y en 1999, Canadá hizo lo mismo. En marzo de 2003, la utilización de rt-PA dentro de las primeras 3 horas después del inicio del accidente cerebrovascular fue aprobado por la Agencia Europea de Medicamentos, a pesar de que estaba condicionada a la fase IV SITS-MOST estudio, que ha sido completado, y los resultados de los cuales fueron publicados recientemente (Wahlgren, y otros, 2005)

Los beneficios reportados se basan en los resultados del estudio NINDS, lo que demuestra que el uso de rt-PA en las primeras 3 horas produjo un aumento absoluto del 11\% al $13 \%$ en el número de pacientes con un resultado excelente. En comparación con los pacientes que recibieron placebo, los pacientes tratados con rt-PA tenían una posibilidad 30\% más alta de ser asintomática o tener 


\section{Consideraciones frente a la gravedad de enfermedades cerebrovasculares}

Vol. 3, núm. 3., (2019)

Leurys Beatriz Moreira Plaza; Viviana del Rocío Moreira Quiroz; Fabián Xavier Moreira

Rivadeneira; Giancarlo Cristóbal Cañarte Baque

una discapacidad mínimo 3 meses después del accidente cerebrovascular. Este beneficio no se acompaña de un aumento de la mortalidad, aunque se asocia con una incidencia de la hemorragia cerebral sintomático de $6,4 \%$, frente al $0,6 \%$ en el grupo placebo.

Este hallazgo enfatiza el hecho de que el tratamiento debe ser administrado por profesionales con una comprensión completa de su uso. La Agencia Europea del Medicamento recomienda su administración por personal con experiencia en el tratamiento del ictus agudo. Un número de estudios prospectivos de fase IV en el que los criterios de tratamiento NINDS (Albers, y otros, 2000)

\section{La trombolisis intraarterial.}

El tratamiento intraarterial con prouroquinasa en oclusión de la arteria cerebral media se ha encontrado para ser seguro y eficaz cuando se administra dentro de las 6 horas de la aparición de los síntomas (estudio PROACT II) (Furlan, Higashida, \& Wechsler, 2009). La FDA no ha aprobado el uso de prouroquinasa porque nunca se llevó a cabo un estudio de confirmación, aunque se utiliza en la práctica clínica para el tratamiento intraarterial de oclusión de la arteria basilar.

La limitación más importante para el tratamiento trombolítico intraarterial es la necesidad de realizar una angiografía supraselectiva de emergencia, que requiere de un neurólogo o un neuroradiólogo intervencionista y un equipo especial atención al ictus. Los nuevos enfoques incluyen la terapia de combinación, con el tratamiento inicial con rt-PA intravenoso en el hospital local, seguida de un rápido traslado al centro de tratamiento del accidente cerebrovascular para la angiografía y la evaluación de los beneficios de la fibrinolisis intraarterial, si recanalización no ha tenido lugar.

\section{Terapia antiplaquetaria y anticoagulante durante la fase aguda}

La administración de aspirina 48 horas después de la ocurrencia de accidente cerebrovascular reduce las tasas de mortalidad y recurrencia, de acuerdo con 2 grandes estudios no cegados.

No hay evidencia científica de los beneficios de la anticoagulación temprana durante la fase aguda del ictus, aunque es una práctica clínica habitual en ciertas situaciones, como recurrente AIT, 


\section{Consideraciones frente a la gravedad de enfermedades cerebrovasculares}

Vol. 3, núm. 3., (2019)

Leurys Beatriz Moreira Plaza; Viviana del Rocío Moreira Quiroz; Fabián Xavier Moreira Rivadeneira; Giancarlo Cristóbal Cañarte Baque

disección arterial, trombosis basilar, y sospecha de accidente cerebrovascular isquémico de origen cardioembólico cuando hay un alto riesgo de reembolización (válvulas artificiales, fibrilación auricular, infarto de miocardio agudo $[\mathrm{AMI}]$ con trombo pared).

En el caso de la trombosis venosa, la profilaxis con siempre se recomienda heparina de bajo peso molecular.

\section{Conclusiones.}

La experiencia acumulada sobre el diagnóstico clínico de la enfermedad cerebrovascular isquémica de origen extracraneal permite ofrecer algunos datos sobre la variedad de manifestaciones clínicas, características demográficas, principales factores de riesgo quirúrgicos y la frecuencia de asociación con lesiones vasculares en otras áreas (aorta abdominal y las arterias de los miembros inferiores) que aseguraron su relación con el pronóstico de la enfermedad.

Se coincide con aquellas publicaciones que plantean la existencia de dos categorías de pacientes: los sintomáticos y los asintomáticos; aunque algunos autores señalan que este planteamiento, el cual descansa en la presencia o no de síntomas hemisféricos, no debería ser tan estricto, ya que en ocasiones existen manifestaciones neurológicas no hemisféricas que son expresión de una llamada isquemia cerebral difusa. Otro resultado importante es el reconocimiento de la localización topográfica de la enfermedad vascular extracraneal, de la que dependerán las manifestaciones clínicas y el pronóstico de la enfermedad. Como conclusión podemos señalar que esta revisión confirmó que la enfermedad cerebrovascular extracraneal es una entidad multicausal donde la estenosis carotídea es la causa más frecuente. El diagnóstico clínico es indispensable en la implementación de la metodología diagnóstica. Desde el punto de vista clínico, los pacientes sintomáticos presentan manifestaciones de isquemia cerebral focal de carácter agudo donde juegan papeles fundamentales: la atención médica del neurólogo, el internista y los especialistas de medicina general integral. La existencia de pacientes asintomáticos exige de la realización de un adecuado interrogatorio y de un examen vascular, sobre todo en aquellos con presencia de factores de riesgo y por último, el tratamiento de la enfermedad cerebrovascular extracraneal debe ser multidisciplinario, en las unidades de ictus, con la opinión del cirujano vascular cuando sea 


\section{Consideraciones frente a la gravedad de enfermedades cerebrovasculares}

Vol. 3, núm. 3., (2019)

Leurys Beatriz Moreira Plaza; Viviana del Rocío Moreira Quiroz; Fabián Xavier Moreira Rivadeneira; Giancarlo Cristóbal Cañarte Baque

necesaria, debido a la eficacia demostrada del tratamiento quirúrgico en pacientes con criterio de selección adecuada en la prevención primaria y secundaria del ictus mayor.

\section{Bibliografía.}

Albers, G., Bates, V., Clark, W., Bell, R., Verro, P., \& Hamilton, S. (2000). Intravenous tissuetype plasminogen activator for treatment of acute stroke: The Standard Treatment with Alteplase to Reverse Stroke (STARS) study. JAMA, 1145-1150.

Arboix, A., Álvarez-Sabín, J., \& Soler, L. (1998). En nombre del Comité de redacción ad hoc del Grupo de estudio de Enfermedades Cerebrovasculares de la SEN. Ictus. Tipos etiológicos y criterios diagnósticos. Neurología. Suppl 3, 4-10.

Bolívar, J. (2015). Investigación Documental. México. Pax.

Castillo, J., \& Martinez-Vila, E. (1995). Trombosis, farmacos antitromboticos y enfermedad cerebrovascular. Barcelona: Uriach \& Cia.

Castro, J. (2016). Técnicas Documentales. México. Limusa.

Davalos, A., Castillo, J., \& Martinez-Vila, E. (1995). Cerebrovascular Disease Study Group of the Spanish Society of Neurology. Delay in neurological attention and stroke outcome. Stroke, 2233-2237.

Davila, A. (2015). Concepto de terminos cientificos. Caracas: Oasis.

Furlan, A., Higashida, R., \& Wechsler, L. (2009). Intra-arterial prourokinase for acute ischemic stroke. The PROACT II study: a randomized controlled trial. Prolyse in Acute Cerebral Thromboembolism. JAMA, 2003-2011.

Johnston, S., Gress, D., Browner, W., \& Sidney, S. (2000). Short term prognosis after emergency department diagnosis of TIA. JAMA., 2901-2906. 
Consideraciones frente a la gravedad de enfermedades cerebrovasculares

Vol. 3, núm. 3., (2019)

Leurys Beatriz Moreira Plaza; Viviana del Rocío Moreira Quiroz; Fabián Xavier Moreira Rivadeneira; Giancarlo Cristóbal Cañarte Baque

Langhorne, P., Willians, B., Gilchrist, W., \& Howie, K. (2003). Do stroke units save lives? Lancet., 395-398.

Powers, W. (2003). Acute hypertension after stroke: the scientific basis for treatment decisions. Neurology, 461-467.

Wahlgren, N., Ahmed, N., Davalos, A., Ford, G., Grond, M., \& Hacke, W. (2005). Thrombolysis with alteplase for acute ischaemic stroke in the Safe Implementation of Thrombolysis in Stroke-Monitoring Study (SITS-MOST): an observational study. Lancet., 798-801.

$$
\text { (c) (1) (9)(2) }
$$

RECONOCIMIENTO-NOCOMERCIAL-COMPARTIRIGUAL

CC BY-NC-SA

ESTA LICENCIA PERMITE A OTROS ENTREMEZCLAR, AJUSTAR Y CONSTRUIR A PARTIR DE SU OBRA CON FINES NO COMERCIALES, SIEMPRE Y CUANDO LE RECONOZCAN LA AUTORÍA Y SUS NUEVAS CREACIONES ESTÉN BAJO UNA LICENCIA CON LOS MISMOS TÉRMINOS. 